\title{
Using Syndromic Surveillance Data to Study the Impact of Media Content on Self-harm
}

\author{
Kristin M. Holland, Francis Annor, Marissa L. Zwald, Jing Wang, Michael Coletta, Aaron Kite \\ Powell, Deborah M. Stone, Steven A. Sumner, Daniel Bowen, ALANA M. VIVOLO-KANTOR, \\ Alex E. Crosby
}

Division of Violence Prevention, Centers for Disease Control and Prevention, Atlanta, Georgia, United States

Objective

To describe national-level trends in nonfatal self-harm and suicidal ideation among 10-19 year old youth from January 2016 through December 2017 and examine the impact of popular entertainment on suicidal behavior.

Introduction

In 2016, a half million people were treated in U.S. emergency departments (EDs) as a result of self-harm [1]. Not only is self-harm a major cause of morbidity in the U.S., but it is also one of the best predictors of suicide. Given that approximately $40 \%$ of suicide decedents visited an ED in the year prior to their death and that the majority of medically-serious self-harm patients are treated in EDs [2], EDs serve as a critical setting in which to monitor rates and trends of suicidal behavior.

To date, the majority of ED data for self-harm are generally two to three years old and thereby can only be used to describe historical patterns in suicidal behavior. Thus, in 2018, a syndrome definition for suicide attempts and suicidal ideation (SA/SI) was de veloped by the International Society for Disease Surveillance (ISDS) Syndrome Definition Committee in conjunction with Centers for Disease Control and Prevention (CDC) staff, allowing researchers to better monitor recent trends in medically treated suicidal behavior using data from the CDC's National Syndromic Surveillance Program (NSSP). These data serve as a valuable resource to help detect deviations from typical patterns of SA/SI and can help drive public health response if atypical activity, such as geospatial or temporal clusters of SA/SI, is observed. Such patterns may be indicative of suicide contagion (i.e., exposure to the suicide or suicidal behavior of a friend or loved one, or through media content, that may put individuals at increased risk of suicidal behavior).

Research has demonstrated that suicide contagion is a real phenomenon [3]. 13 Reasons Why is a Netflix series focused on social, school, and family-related challenges experienced by a high school sophomore; each episode in the 13-episode series describes a problem faced by the main character, which she indicates contributed to her decision to die by suicide. The series premiered March 31, 2017 and is rated TV-MA by TV Parental Guidelines [4] (may be unsuitable for those under age 18 years due to graphic content). Nevertheless, the series has become popular among youth under 18 years of age. Of note, in the final episode, the main character's suicide by wrist laceration is graphically depicted. Following the premiere of the series, researchers and psychologists across the U.S. expressed concern that this graphic depiction of suicide could result in a contagion effect, potentially exacerbating suicidal thoughts and behavior among vulnerable youth viewers. To date, the only empirical data demonstrating the potential iatrogenic effects of this graphic portrayal of suicide comes from a study of Google Trends data demonstrating an increase in online suicide queries in the weeks following the show, with most of the queries focusing on suicidal ideation (e.g., "how to commit suicide," "how to kill yourself") [5]. However, there has been no study to examine changes in nonfatal self-harm trends following the series debut.

\section{Methods}

NSSP data were aggregated at the national level from January 2016 through December 2017 to examine weekly trends in the percentage of ED visits that involved SA/SI among all ED visits for youth aged 10-19. Google Trends data were also used to examine suicide-related online searches conducted during this period. Additional sensitivity analyses to explore these findings will be conducted by HHS region using NSSP data. 


\section{Results}

Preliminary results suggest an increase in ED visits due to SA/SI among 10-19 year old youth across the U.S. beginning about two weeks after the premiere of 13 Reasons Why (April 16, 2017) and lasting a total of six weeks before weekly percentages of SA/SI ED visits returned to their endemic levels during the week of May 28-June 3, 2017. The peak of the increase represented a 26\% increase in SA/SI compared to the highest weekly percentage of these visits in the previous 15 weeks in 2017. Additionally, this peak coincided with marked peaks in online searches for phrases including "13 Reasons Why" from March 26-June 3, 2017, "how to kill yourself" from April 16-June 3, 2017, and "how to slit wrists" from April 2-June 3, 2017 as demonstrated by Google Trends data.

\section{Conclusions}

This study demonstrates the utility of syndromic surveillance data for tracking SA/SI at the national level and for detecting atypical fluctuations in trends over time. Using syndromic surveillance data for this purpose could help spark public health response to emerging health threats. However, it is important to note that NSSP data are subject to some limitations; for instance, although about $60 \%$ of ED visits in the U.S. are captured in NSSP, the system is not nationally representative and thus, these findings are not generalizable to areas not participating in NSSP. Additionally, our definition may under- or over-estimate SA/SI based on differences in chief complaints or discharge diagnosis data between jurisdictions. Further, hospital participation in NSSP can vary across months-a factor that could contribute to trends observed in NSSP data. Finally, these analyses explored the concurrent trends in SA/SI among youth and the popularity of only one television series. Although these analyses point to an association between the increases in SA/SI and the time period in which the series reached its peak popularity as evidenced by Google Trends, there may have been other sociocultural factors that impacted SA/SI trends during the study period. Still, preliminary findings suggest that media content containing graphic depictions of suicide viewed by youth audiences may contribute to increases in ED visits for selfharm and suicidal ideation, as well as greater interest in searching for information about suicidal behavior online. While it is impossible to assess causation, these results are consistent with the phenomenon of suicide contagion. It is also possible that the series or related media coverage during this time increased help-seeking among some youth or their families that contributed to the increases observed. Regardless of the underlying mechanism, entertainment content creators may consider referring to the Recommendations for Reporting on Suicide (www.reportingonsuicide.org), which can help reduce the risk of suicide among vulnerable individuals and avoid contributing to suicide contagion while promoting suicide prevention messages. Finally, ongoing surveillance of suicidal behavior using NSSP data could help reduce the burden of nonfatal self-harm by catalyzing the implementation of prevention efforts.

\section{References}

1. Center for Disease Control and Prevention, National Center for Injury Prevention and Control. (2018). Webbased Injury Statistics Query and Reporting System (WISQARS). Available from www.cdc.gov/ncipc/wisqars. Accessed 10-3-2018.

2. Ahmedani BK, Simon GE, Stewart C, et al. 2014. Health care contacts in the year before suicide death. $J$ Gen Intern Med. 29, 870-77. PubMed https://doi.org/10.1007/s11606-014-2767-3

3. Gould M, Jamieson P, Romer D. 2003. Media contagion and suicide among the young. Am Behav Sci. 46(9), 1269-84. https://doi.org/10.1177/0002764202250670

4. The TV Parental Guidelines. (2018). Available from http://tvguidelines.org/. Accessed 10-3-2018.

5. Ayers JW, Althouse BM, Leas EC, Dredze M, Allem JP. 2017. Internet searches for suicide following the release of 13 Reasons Why. JAMA Intern Med. 177(10), 1527-29. PubMed

https://doi.org/10.1001/jamainternmed.2017.3333 